\title{
Zambomballooning: A novel technique to seal a coronary perforation complicating the intervention of a chronic total occlusion
}

\author{
Juan Luis Gutiérrez-Chico ${ }^{1,2}$ \\ ${ }^{1}$ Head of Interventional Cardiology, DRK-Klinikum Westend, Berlin, Germany \\ ${ }^{2}$ Institute of Cardiovascular Translational Research of Atlantic (ICTRA), Berlin, Germany
}

This paper was guest edited by Prof. Kambis Mashayekhi

\begin{abstract}
Zambomballooning, a novel technique to seal a coronary perforation is hereby described with a representative case. A 71-year-old male patient with stable angina and positive scintigraphy underwent antegrade percutaneous coronary intervention of chronic total occlusion in the proximal left circumflex artery (Fig. 1A, Suppl. Video 1 - see journal website). A GAIA-3 wire crossed distally, supported by a CARAVEL microcatheter (Asahi Intecc, Nagoya, JP), but the microcatheter had difficulties to follow and no blood could be aspirated distally, thus suggesting that the wire had exited the vessel. When the microcatheter was removed, a coronary perforation could be noticed (Fig. 1B, Suppl. Videos 2, 3 - see journal website), with extravasation of contrast into the pericardium (Fig. 1B, red arrows). A rapid-exchange balloon was inflated proximally to achieve haemostasis. The wire was withdrawn to the distal edge of the balloon (Fig. 1C, Suppl. Video 4 - see journal website), resembling a zambomba, (Fig. 1D) and used to re-entry the true lumen, taking advantage of the support provided by the haemostasis balloon. Once the wire lay distally in the true lumen, the haemostasis balloon was exchanged for predilation, and the predilation had the effect of sealing the perforation definitely (Fig. 1E, Suppl. Videos 5, 6 - see journal website). A plausible mechanism for this phenomenon is
\end{abstract}

proposed in Figure 2. Optimal angiographic (Fig. 1F, Suppl. Video 7 - see journal website) and clinical result could be achieved. The patient was discharged $24 \mathrm{~h}$ later, asymptomatic and without pericardial effusion in echo.

Hemostasis of a coronary perforation by means of prolonged balloon inflation has been previously described [1]. The novelty of zambomballooning lies on making the most of the superb backup of the haemostasis balloon to regain the true lumen with a high-penetrance wire. This technique allows predilation to seal naturally the perforation, avoiding pericardiocentesis, surgery or covered stents with high restenosis rates. If the perforation is noticed with a wire in suboptimal condition for re-entry, haemostasis with an over-the-wire balloon can be preferred, so the wire can be exchanged $(\geq 7 \mathrm{~F}$ guiding catheter recommended). The technique is named after a folk music instrument for Christmas carols, called zambomba in Spanish or Brummtopf in German (Fig. 1D).

\section{Conflict of interest: None declared}

\section{Reference}

1. Gruberg L, Pinnow E, Flood R, et al. Incidence, management, and outcome of coronary artery perforation during percutaneous coronary intervention. Am J Cardiol. 2000;86(6): 680-2, A8.

Address for correspondence: Prof. Juan Luis Gutiérrez-Chico, MD, PhD, FESC, FACC, Head of Interventional Cardiology, DRK-Klinikum Westend, Spandauer Damm 130, 14050 - Berlin, Germany, tel: +49 (0) 176 30585019, +34 615 319370 , e-mail: juanluis.gutierrezchico@ictra.es

Received: 15.08.2017 Accepted: 03.09.2017 


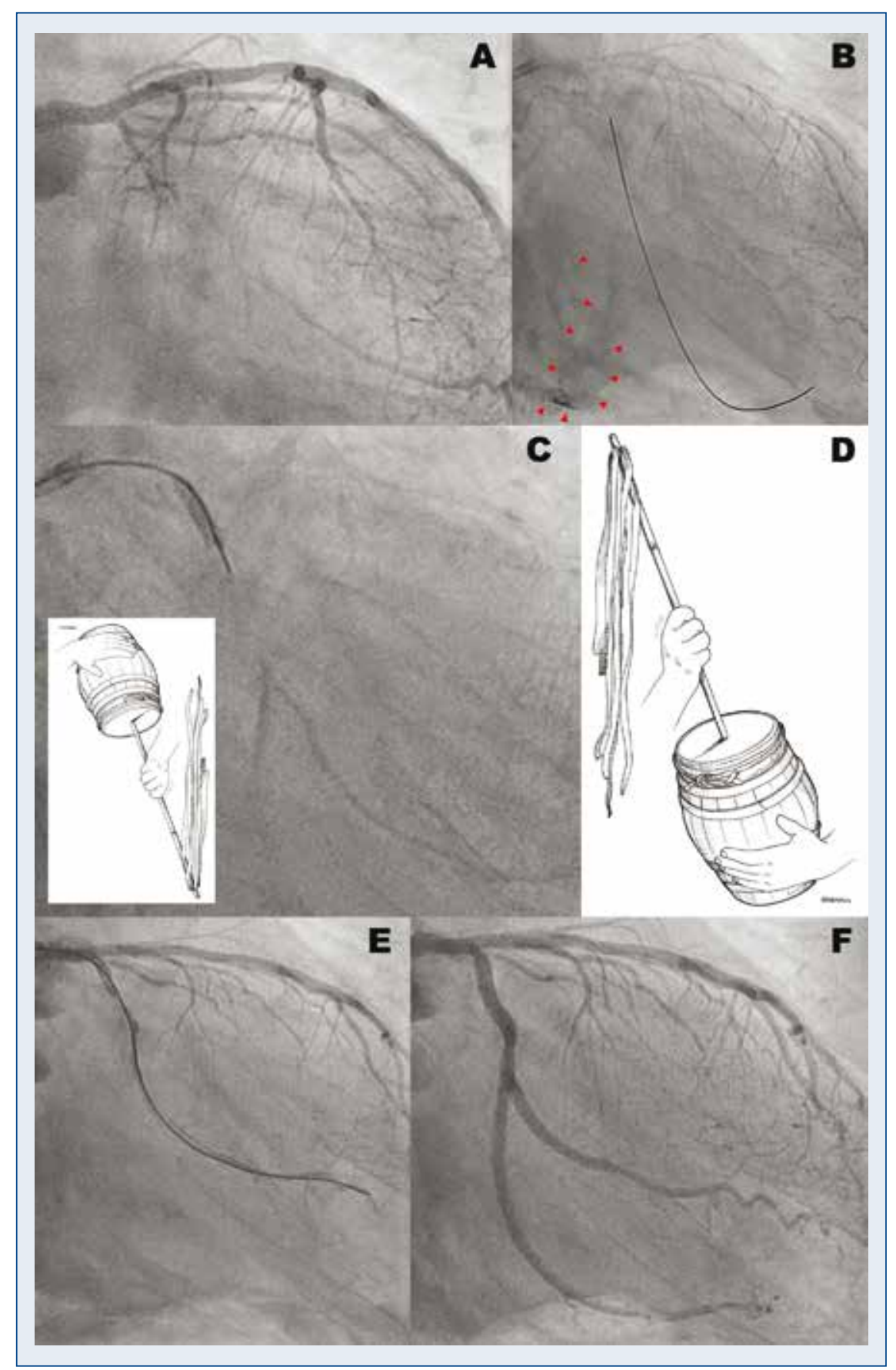

Figure 1. Representative case of a coronary perforation sealed with zambomballooning technique; A. Chronic total occlusion of the proximal left circumflex artery, with retrograde collateral filling; B. Coronary perforation bleeding into the pericardium; C. Zambomballooning: prolonged proximal balloon inflation to achieve hemostasis; the wire is pulled back to the distal edge of the balloon and used to re-enter into the true lumen; D. Zambomba (Spanish) or Brummtopf (German); E. Coronary perforation is definitely sealed after predilation with the wire placed distally in the true lumen; F. Optimal final angiographic result. 


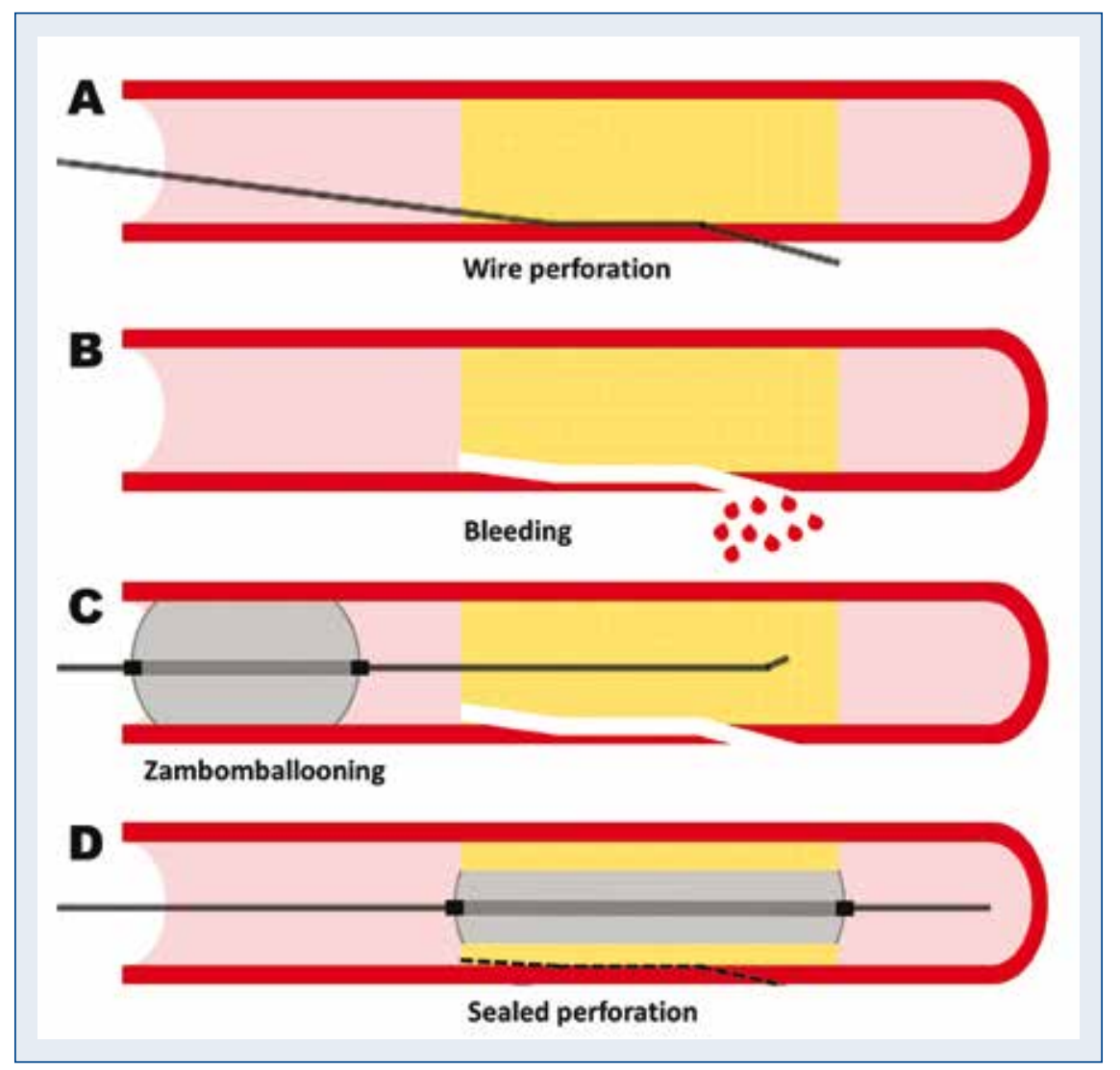

Figure 2. Plausible mechanism for the sealing effect of zambomballooning; A. Wire perforation, following an acute angulation with respect to the axis of the vessel; B. If a microcatheter is advanced distally, or if predilation is performed, the perforation results in significant bleeding. In case of intrapericardial bleeding, this evolves quickly into tamponade if no action is taken; C. Zambomballooning: Proximal balloon inflation to achieve haemostasis; the wire is then pulled back to the distal edge of the balloon and used to re-enter the true lumen, making the most of the superb back-up of the haemostasis balloon; D. When the wire lies distally in the true lumen, the predilation obturates the angulated perforation channel and the plaque shift acts as a natural plug, thus definitely sealing the perforation, without the need of any additional device. 